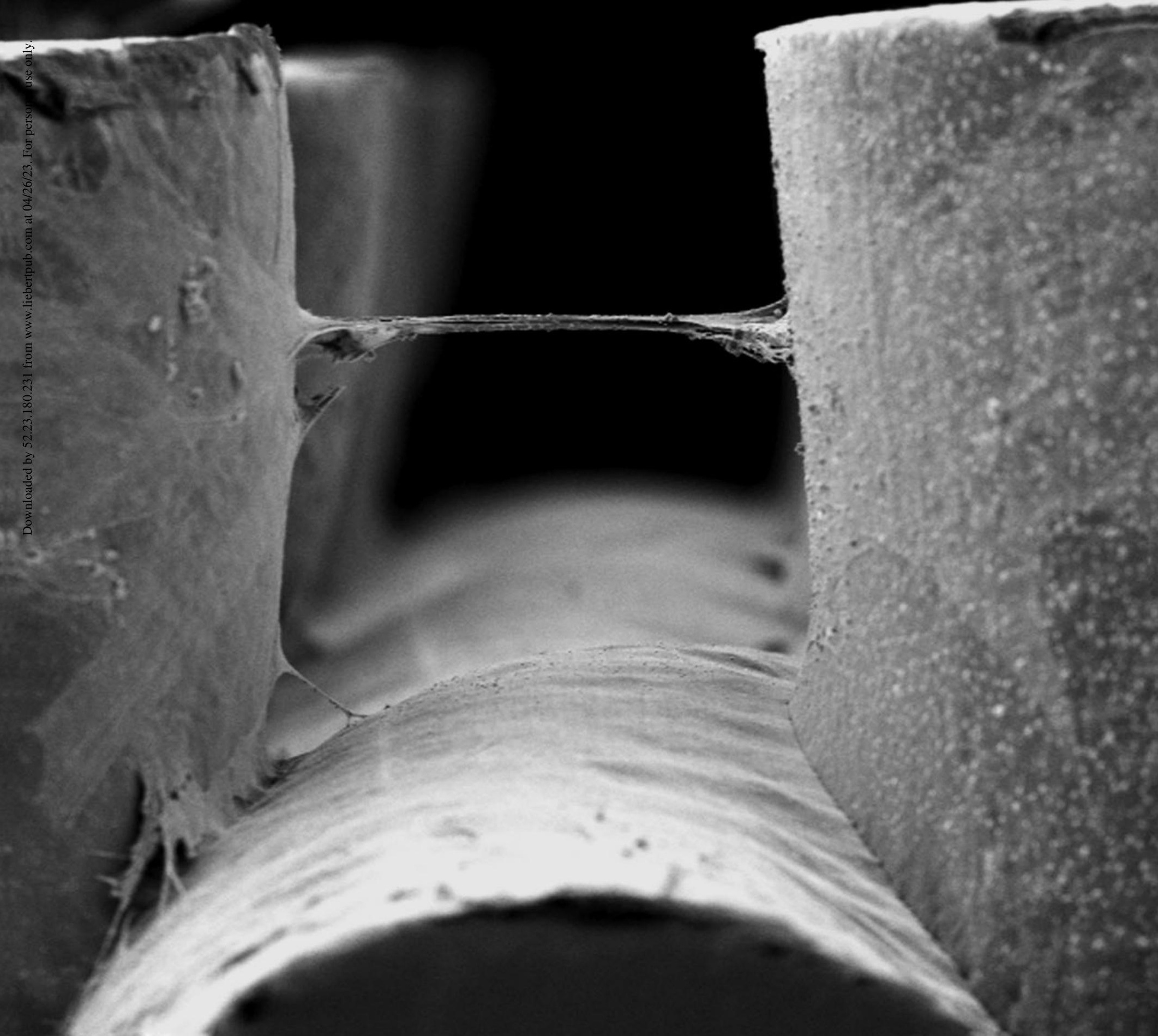




\title{
3D-Printed Poly( $\varepsilon$-caprolactone)/Graphene Scaffolds Activated with P1-Latex Protein for Bone Regeneration
}

\author{
Guilherme Ferreira Caetano,,2,* Weiguang Wang,, ${ }^{1, *}$ Wei-Hung Chiang, ${ }^{3}$ Glen Cooper, \\ Carl Diver, ${ }^{1}$ Jonny James Blaker, ${ }^{4}$ Marco Andrey Frade, and Paulo Bártolo ${ }^{1}$
}

\begin{abstract}
Biomanufacturing is a relatively new research domain focusing on the use of additive manufacturing technologies, biomaterials, cells, and biomolecular signals to produce tissue constructs for tissue engineering. For bone regeneration, researchers are focusing on the use of polymeric and polymer/ceramic scaffolds seeded with osteoblasts or mesenchymal stem cells. However, high-performance scaffolds in terms of mechanical, cell stimulation, and biological performance are still required. This article investigates the use of an extrusion additive manufacturing system to produce poly( $\varepsilon$-caprolactone) (PCL) and PCL/graphene nanosheet scaffolds for bone applications. Scaffolds with regular and reproducible architecture and uniform dispersion of graphene were produced and coated with P1-latex protein extracted from the Hevea brasiliensis rubber tree. Results show that the obtained scaffolds cultivated with human adipose-derived stem cells present no toxicity effects. The presence of graphene nanosheet and P1-latex protein in the scaffolds increased cell proliferation compared with PCL scaffolds. Moreover, the presence of P1-latex protein promotes earlier osteogenic differentiation, suggesting that PCL/graphene/P1-latex protein scaffolds are suitable for bone regeneration applications.
\end{abstract}

Keywords: biofabrication, graphene, P1-latex protein, scaffold, tissue engineering

\section{Introduction}

TISSUE ENGINEERING is recognized as a promising field to overcome some of the limitations of existing clinical treatments for the repair of damaged and dysfunctional tissues or organs. ${ }^{1-3}$ A key strategy involves the use of biocompatible and biodegradable materials, cells, and growth factors combined with additive manufacturing techniques to produce tissue constructs. ${ }^{1,4-6}$ Two main approaches are usually considered. ${ }^{7-9}$ The first approach is based on the use of biodegradable and biocompatible porous scaffolds that are implanted into the lesion site without cells, seeded with autologous or allogeneic cells and then implanted, or seeded with cells and then cultured in vitro to produce tissue-engineered constructs prior implantation. ${ }^{6-10}$ In this approach, scaffolds, from either natural or synthetic materials, provide the appropriated biomechanical environment to allow cells to produce their own extracellular matrix. In the second approach, specific additive manufacturing techniques (e.g., extrusion-based processes, laser bioprinting, or material jetting processes) are used to print cells immobilized within polymeric hydrogels producing cell-laden three-dimensional (3D) constructs. ${ }^{6-8,11,12}$

Synthetic polymers [e.g., poly(glycolic acid), poly(lactic acid), poly(e-caprolactone) (PCL), poly(lactide-co-glycolide)] are the most commonly used materials for certain applications such as bone and osteochondral tissue engineering. ${ }^{13-15}$

\footnotetext{
${ }^{1}$ Manchester Biomanufacturing Centre, School of Mechanical, Aerospace and Civil Engineering, University of Manchester, Manchester, United Kingdom.

${ }^{2}$ Department of Internal Medicine, Ribeirão Preto School of Medicine, University of São Paulo, São Paulo, Brazil.

${ }^{3}$ Department of Chemical Engineering, National Taiwan University of Science and Technology, Taipei, Taiwan.

${ }^{4}$ Bio-Active Materials Group, School of Materials, MSS Tower, The University of Manchester, Manchester, United Kingdom.

*Cofirst authors, contributed equally to this work.

Opposite page: Scanning electron micrograph showing human adipose-derived stem cells bridging through two filaments of a 3D printed polymer/graphene scaffold. Photo credit: Guilherme Ferreira Caetano, Weiguang Wang, Wei-Hung Chiang, Glen Cooper, Carl Diver, Jonny James Blaker, Marco Andrey Frade, and Paulo Bártolo.
} 
Among these polymers, PCL is widely used due to its biocompatibility and favorable rheological properties, including low glass transition temperature (approximately $-60^{\circ} \mathrm{C}$ ), low melting point $\left(\sim 60^{\circ} \mathrm{C}\right)$, and decomposition temperature of around $350^{\circ} \mathrm{C}$, which facilitate its printability. ${ }^{16-18}$ However, PCL does not provide specific motifs for cell attachment and is highly hydrophobic, which compromises its bioactivity and does not possess the required strength to match the mechanical properties of bone.

To improve the performance of PCL scaffolds, this article investigates the use of a screw-assisted extrusionbased additive manufacturing system to produce totally novel 3D PCL/graphene/P1-latex protein-coated scaffolds for bone regeneration. Graphene is used to improve the mechanical properties of the scaffolds and to impact the biological properties due to its high surface area, stiffness, and the presence of wrinkles and ripples created during the production of graphene. Composite scaffolds with low graphene contents are considered to minimize potential cytotoxicity risks. The P1-latex protein extracted from the Hevea brasiliensis rubber tree is used to improve the biological performance of produced scaffolds. Preliminary results showed that latex biomembranes can be successfully used for wound healing applications, enabling cell adhesion, and stimulating various cell types involved in the healing process. ${ }^{19-23}$ These biomembranes were also used to treat critical size bone defects created in rabbit's calvaria. ${ }^{21}$

$\mathrm{PCL} /$ graphene scaffolds containing different quantities of graphene nanosheets were printed and coated by soaking them in the P1-latex protein solution. The biological performance of these scaffolds was assessed using human adipose-derived stem cells (hADSCs) for both cell viability/ proliferation and cell differentiation tests.

\section{Materials and Methods}

\section{Materials and scaffold fabrication}

PCL, Capa $6500(\mathrm{Mw}=50,000)$, was purchased from Perstorp (UK). Graphene nanosheets were prepared by a water-assisted liquid phase exfoliation of graphite as reported previously. ${ }^{24,25}$ Lyophilized P1 latex protein from the $H$. brasiliensis rubber tree was purchased from PeleNova Biotecnologia (Brazil). PCL/graphene composite blends were prepared by melt blending. In this case, PCL pellets were heated $>70^{\circ} \mathrm{C}$ in a bowl to ensure that all material is in a molten state before graphene addition. PCL and graphene were mixed for $15 \mathrm{~min}$ to guarantee a homogeneous dispersion. After cooling for $2 \mathrm{~h}$, the blended material was cut into small pellets. The exact amount of graphene present in the mixtures was determined using a TA Instruments Q500 TGA. Thermogravimetric analysis was performed using PCL as control and PCL/graphene blends. Scans were performed in an air atmosphere (flow at $60 \mathrm{~mL} / \mathrm{min}$ ) with a temperature ranging from room temperature to $560^{\circ} \mathrm{C}$ at a rate of $10^{\circ} \mathrm{C} / \mathrm{min}$.

Scaffolds containing different concentrations of graphene $(0.13,0.50$, and $0.78 \mathrm{wt} . \%)$ were produced using a screwassisted additive manufacturing system from RegenHU (3DDiscovery, Switzerland) (Fig. 1a). Scaffolds were produced using a $0^{\circ} / 90^{\circ}$ lay down pattern and the following process parameters: $90^{\circ} \mathrm{C}$ of melting temperature, $220 \mu \mathrm{m}$ of slice thickness, $22 \mathrm{rpm}$ of screw rotation velocity, and $20 \mathrm{~mm} /$ $\mathrm{s}$ of deposition velocity. A nozzle of $330 \mu \mathrm{m}$ of diameter and a constant filament distance of $680 \mu \mathrm{m}$ were also considered.

After fabrication, the scaffolds were cut into small blocks to fit into the wells of a 24 -well plate $(11 \times 11 \times 6 \mathrm{~mm})$, sterilized in $70 \%$ ethanol, prepared with sterile water, and then P1-latex protein adsorption, before soaking them in the

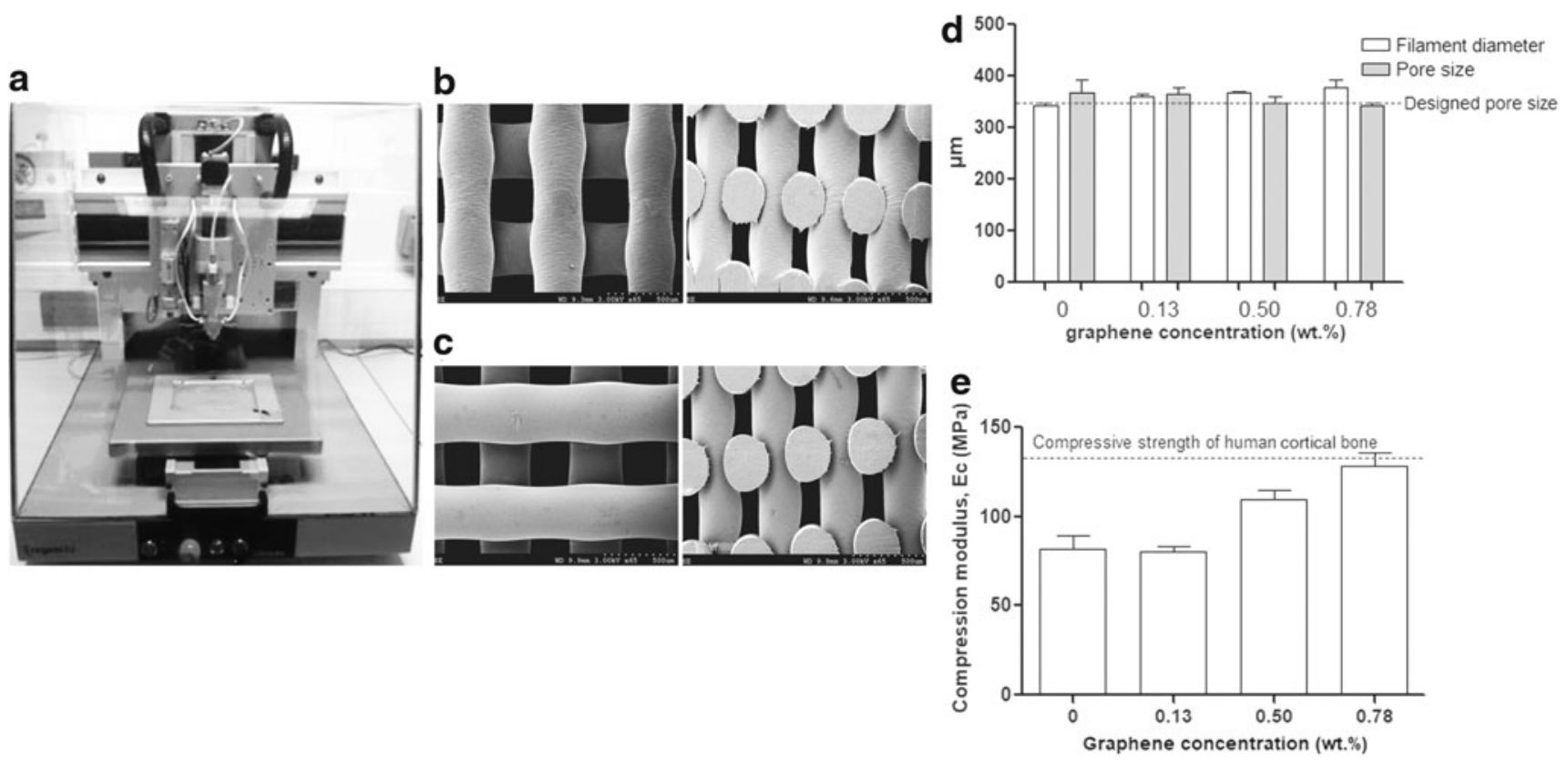

FIG. 1. (a) Screw-assisted additive manufacturing system from RegenHU (3DDiscovery, Switzerland); (b) SEM images of top and cross section view of PCL scaffolds; (c) SEM images of top and cross section view of 0.78 wt.\% PCL/graphene scaffolds; (d) morphological data (filament diameter and pore size) of PCL and PCL/graphene scaffolds; (e) mechanical characterization (compressive modulus) of PCL and PCL/graphene scaffolds. PCL, poly( $\varepsilon$-caprolactone); SEM, scanning electron microscopy. 
cell culture medium, basal medium (MesenPRO RS ${ }^{\mathrm{TM}}$ Basal Media; Thermo Fisher Scientific, Waltham, MA). P1-latex protein was analyzed by Limulus Amebocyte Lysate (LAL, from LONZA), a standard endpoint assay for detection of liposaccharides (LPS), Gram-negative bacterial endotoxin, presenting LPS levels below the limited point.

\section{Scanning electron microscopy}

To assess the morphology of fabricated scaffolds, thin top and cross section layers of each scaffold sample (around $3 \mathrm{~mm}$ ) were cut and gold/palladium coated for imaging using a Q150T turbopumped sputter coater (Quorum Technologies, UK) to an approximate thickness of $10 \mathrm{~nm}$. Scanning electron microscopy (SEM) images were obtained using a Hitachi S3000N scanning electron microscope (Hitachi, Maidenhead, UK). Corresponding parameters were measured through ImageJ software (NIH, Bethesda, MD) on the obtained SEM images.

\section{Mechanical characterization}

Compression tests were performed to assess the effect of different graphene concentrations on the mechanical properties of the scaffolds. Blocks of $5.0 \mathrm{~mm}$ in length, $5.0 \mathrm{~mm}$ in width, and $5.72 \mathrm{~mm}$ in height $\left(\mathrm{h}_{0}\right)$ were considered. Tests were carried out on scaffolds in dry state at a rate of $1 \mathrm{~mm} /$ min up to a strain limit of $0.3 \mathrm{~mm} / \mathrm{mm}(30 \%)$, using the INSTRON 4507 testing system equipped with a $1 \mathrm{kN}$ load cell. During the uniaxial compression tests, the software determined force $\mathrm{F}$ and corresponding displacement values, which were converted into engineering stress $\sigma$ and strain $\varepsilon$ as follows: $\sigma=\frac{F}{A}, \varepsilon=\frac{\Delta h}{h_{0}}$, where A is the initial sample cross section area and $\Delta h$ is the scaffold height variation. The obtained stress-strain data were further processed to determine the compressive modulus, $E$, according to the procedure reported by Fiedler $e t a l .{ }^{26}$

\section{Graphene dispersion analysis}

Ex situ characterization of the graphene dispersion was performed by transmission electron microscopy (TEM) and micro-Raman spectroscopy. Spherical aberration corrected field emission TEM (ARM-200F; JEOL, Tokyo, Japan) with $200 \mathrm{kV}$ accelerating voltage was used. The TEM samples were prepared by dispersing few drops of graphene dispersion onto TEM copper microgrids (400 mesh; Ted Pella, Redding, CA) and then drying in ambient conditions. Raman scattering studies were performed at room temperature using a JASCO 5100 (JASCO, Tokyo, Japan) spectrometer $(\lambda=533 \mathrm{~nm})$. Spectra were averaged from 10 random positions on each sample.

\section{P1-latex protein adsorption}

After sterilization, scaffolds were rinsed twice with phosphate buffer saline (PBS) and dried for $24 \mathrm{~h}$ in a sterile $37^{\circ} \mathrm{C}$ incubator environment to remove any remaining ethanol and PBS. The scaffolds were then adsorbed with P1-latex protein using a procedure described by Patel et al. to coat PCL substrates. ${ }^{27}$ In brief, lyophilized P1-latex protein was prepared in $1 \mathrm{~mL}$ Dulbecco's phosphate-buffered saline (DPBS) to obtain $1 \%(\mathrm{w} / \mathrm{v})$ stock, with a concentration of $0.01 \mathrm{~g} / \mathrm{mL}$.
The stock solution was further diluted to the desired concentration of $0.05 \%(\mathrm{w} / \mathrm{v})$ in DPBS and filter sterilized using a $0.22-\mu \mathrm{m}$ filter. Scaffolds were then exposed to $1 \mathrm{~mL}$ of $0.05 \%(\mathrm{w} / \mathrm{v}) \mathrm{P} 1$ solution at $4^{\circ} \mathrm{C}$ for $24 \mathrm{~h}(n=3)$. The amount of protein adsorbed after $24 \mathrm{~h}$ was determined by quantifying the remaining P1-latex protein present in the solution using the Micro BCA ${ }^{\mathrm{TM}}$ Protein Assay Kit (Thermo Scientific, Waltham, MA).

\section{Scratch assay}

For preliminary assessment of the effect of P1-latex protein on cell spreading and cell migration, a scratch assay was conducted following a procedure previously reported. ${ }^{28}$ The cells were seeded into 24 -well tissue culture plates at a concentration of $4 \times 10^{4}$ cells per well and incubated overnight to allow cell attachment and nearly confluent cell monolayers. A linear wound was created on the monolayer using a sterile $200 \mu \mathrm{L}$ plastic pipette tip. Any cellular debris was removed by washing the wells with PBS. A $1 \mathrm{~mL}$ cell culture medium was added according to each group: medium with dimethyl sulfoxide (50\%), as negative control group; basal medium as basal control group; and three concentrations of $\mathrm{P} 1(0.01 \mu \mathrm{g}, 1 \mu \mathrm{g}$, and $100 \mu \mathrm{g}) \mathrm{di}-$ luted in medium.

After $24 \mathrm{~h}$ of incubation, cells were fixed with $3.7 \%$ paraformaldehyde for $15 \mathrm{~min}$ and stained with 4',6-diamino2-phenylindole (DAPI from Invitrogen, USA) for $20 \mathrm{~min}$. Twelve representative images from each group were photographed to estimate the relative cell migration. The data were analyzed using ImageJ software, and the percentage of cell migration was calculated as [cells (basal medium with P1) cells (basal control)/cells (basal control) $] \times 100 \%$.

\section{Cell seeding}

In vitro tests were performed using hADSCs (STEM$\mathrm{PRO}^{\circledR}$; Thermo Fisher Scientific). Cells were cultured in T75 tissue culture flasks (Sigma-Aldrich, Dorset, UK) with MesenPRO RS Basal Media until 80\% confluence and harvested using $0.05 \%$ trypsin-EDTA solution (Thermo Fisher Scientific). After cell counting, cells were seeded on the scaffolds (100 $\mu \mathrm{L}$ of medium containing $\sim 5 \times 10^{4}$ cells per sample), and the cell-seeded scaffolds were incubated at standard conditions $\left(37^{\circ} \mathrm{C}\right.$ under $5 \%$ carbon dioxide and $95 \%$ humidity) for $4 \mathrm{~h}$ to allow cell attachment, before the addition of $1 \mathrm{~mL}$ fresh basal medium.

\section{Cell viability/proliferation}

Cell viability/proliferation was assessed at 7, 14, and 21 days after cell seeding using the Resazurin assay (reagents from Sigma-Aldrich). ${ }^{29,30}$ The medium was changed every 3 days. At each time point, the cell-seeded scaffolds were placed in a new 24-well plate and $1 \mathrm{~mL}$ Alamar Blue solution $0.001 \%(\mathrm{v} / \mathrm{v})$ in culture medium was added to each well. The plates were incubated for $4 \mathrm{~h}$ under standard conditions. After incubation, $150 \mu \mathrm{L}$ of each sample was transferred to a 96well plate and the fluorescence intensity was measured at $540 \mathrm{~nm}$ excitation wavelength and $590 \mathrm{~nm}$ emission wavelength with a spectrophotometer (Sunrise, Tecan, Männedorf, Zurich, Switzerland). Cell proliferation experiments were performed three times in triplicate. 


\section{Cell differentiation}

To determine the osteogenic differentiation of hADSCs cultured in the scaffolds, in basal medium and also in osteogenic medium (StemPro Osteocyte/Chondrocyte Differentiation Basal Media; Thermo Fisher Scientific), alkaline phosphatase (ALP) enzyme activity was quantified using a colorimetric assay (SensoLYTE $^{\circledR}$ pNPP Alkaline Phosphatase Assay Kit), according to the manufacturer. Cell/scaffold constructs were washed with buffer solution, incubated in $0.2 \%(\mathrm{v} / \mathrm{v})$ Triton-X in buffer solution for $10 \mathrm{~min}$, sonicated twice for $2 \mathrm{~min}$, and incubated at $-20^{\circ} \mathrm{C}$ for $10 \mathrm{~min}$. Cell lysates were collected, centrifuged at $300 \mathrm{~g}$ for $5 \mathrm{~min}$ at $4^{\circ} \mathrm{C}$, and $50 \mu \mathrm{L}$ of the cell lysate supernatant was transferred to a 96-well plate and incubated with $50 \mu \mathrm{L}$ of pNPP ALP substrate ( $p$-nitrophenyl phosphate). After $40 \mathrm{~min}$, the absorbance was measured using a plate reader (Sunrise, Tecan) at $405 \mathrm{~nm}$.

Osteogenic differentiation tests were performed three times in triplicate. ALP is reported as mean value \pm standard deviation (SD).

\section{Cell morphology}

Cell-seeded scaffolds were observed with SEM. Sample preparation was performed in accordance with the standards for cell viability/proliferation (21 days) and differentiation tests (28 days). In brief, scaffolds were fixed with $2.5 \%(\mathrm{v} / \mathrm{v})$ glutaraldehyde solution (Sigma-Aldrich) for $30 \mathrm{~min}$ at room temperature, rinsed twice in PBS, then dehydrated with a graded ethanol series $(50 \%, 70 \%, 80 \%, 90 \%$, and $100 \%$, then 50:50 ethanol-hexamethyldisilazane (HMDS) (SigmaAldrich), and $100 \%$ HMDS with 10 min exposure at each step), and then air dried to remove residual HMDS. Thin cross section layers of each sample (around $2 \mathrm{~mm}$ ) were cut to size and then platinum coated for imaging (Gatan Model 682 Precision Etching Coating System, approximate coating thickness $10 \mathrm{~nm}$ ). SEM images were obtained using a Hitachi S3000N microscope (Hitachi).

Cell morphology and cell spreading were further assessed using laser confocal microscopy. Samples were prepared in accordance with the standards for cell viability/proliferation (21 days) and differentiation tests (28 days). Cell membranes and nuclei were stained by removing samples from the cell culture plate, rinsed twice in PBS, and fixed using 4\% (v/v) paraformaldehyde for $40 \mathrm{~min}$. Samples were washed twice with PBS before immersion for $30 \mathrm{~min}$ in an immunocytochemistry blocking buffer comprising $2 \%(\mathrm{w} / \mathrm{v})$ goat serum (31872; Thermo Fisher Scientific) and 1\% (w/v) bovine serum albumin (37525; Thermo Fisher Scientific) in PBS.

Samples were again rinsed twice with PBS. Cell nuclei were stained blue by soaking in a PBS solution containing Hoescht 33342 (C62249; Thermo Fisher Scientific) at a $2 \mu \mathrm{M}$ concentration, whereas cell membranes were stained using CellMask ${ }^{\mathrm{TM}}$ Orange plasma membrane stain (C10045; Thermo Fisher Scientific). Samples were left in the staining solution for $10 \mathrm{~min}$ before removal, rinsed twice with PBS, and mounted using ProLong ${ }^{\circledR}$ Diamond Antifade (P36962; Thermo Fisher Scientific) on glass cover slips. Confocal images were obtained using a Leica TCS SP5 (Leica, Milton Keynes, UK) confocal microscope.

\section{Data analysis}

Data are represented as mean $\pm \mathrm{SD}$. Data were subjected to analysis of variance (ANOVA), one-way ANOVA, fol- lowed by post hoc Tukey's test and two-way ANOVA, followed by post hoc Bonferroni test, depending on the number of variables to analyze. The first statistical test is applied when only one variable is considered, in this case concentration, whereas the second method is applied in cases wherein more than one variable is considered, in this case concentration and time. Significance levels were set at $p<0.05$. GraphPad Prism software was used for statistical analysis and graphing.

\section{Results}

\section{Scaffold characterization}

Printed scaffolds present uniformly distributed regular pores (Fig. 1b, c) with key geometric characteristics indicated in Figure 1d. The addition of graphene nanosheets to PCL showed no significant influence on the filament diameter and pore sizes compared with original designed geometric characteristics ( $330 \mu \mathrm{m}$ filament diameter and $350 \mu \mathrm{m}$ pore size).

Mechanical compression results are presented in Figure 1e. Results show that by increasing the concentration of graphene from 0.13 to $0.78 \mathrm{wt} . \%$, the compressive modulus increased from $80 \mathrm{MPa}$ to around $130 \mathrm{MPa}$. In comparison with PCL scaffolds, a slight decrease in mechanical properties was observed for scaffolds containing $0.13 \%$ pristine graphene that the authors hypothesize could be due to the stress concentration in the interface between the polymer and the pristine graphene, which superpose the reinforcement effect of pristine graphene and the reduction on the pore size. As expected, the mechanical behavior of the scaffolds is strongly correlated with the number of graphene nanosheets incorporated in the PCL matrix. Moreover, maximum values of compressive modulus observed for scaffolds containing higher concentrations of graphene are in the midrange of properties reported for human cortical bone. ${ }^{31}$

\section{Graphene distribution}

TEM was performed to determine the morphology of the prepared graphene nanosheets (Fig. 2a). Results show exfoliated graphene with lateral size ranging between 1500 and $2000 \mathrm{~nm}$ and a structure consisting of few layers. The prepared graphene nanosheets showed three typical peaks assigned as D, G, and 2D peaks at 1340,1577 , and $2692 \mathrm{~cm}^{-1}$, respectively (middle in Fig. 2b).

Moreover, the Raman spectrum of PCL shows several characteristic Raman peaks of PCL at 1438 and $2927 \mathrm{~cm}^{-1}$. The Raman spectrum of PCL/graphene composite also exhibits the $\mathrm{D}, \mathrm{G}$, and $2 \mathrm{D}$ bands of graphene, indicating the presence of graphene in the PCL/graphene scaffold.

Micro-Raman spectroscopy was used to assess the graphene dispersion in the composite scaffolds. Figure $2 \mathrm{c}$ shows the region of PCL/graphene scaffold with $0.50 \mathrm{wt}$.\% graphene considered for the Raman mapping study. Figure $2 \mathrm{~d}$ shows a high magnification image of the PCL/graphene scaffold highlighted in red in the SEM image (Fig. 2c). The image shows the smooth surface of the PCL/graphene scaffold. Figure $2 \mathrm{e}-\mathrm{h}$ presents the corresponding two-dimensional micro-Raman mapping of the $\mathrm{PCL} /$ graphene scaffolds with different graphene concentrations from $0,0.13,0.50$, and $0.78 \mathrm{wt} . \%$.

The false color maps were generated by scanning a $532 \mathrm{~nm}$ laser beam over an area of $250 \times 450 \mu \mathrm{m}^{2}$ in $5 \mu \mathrm{m}$ steps. The 

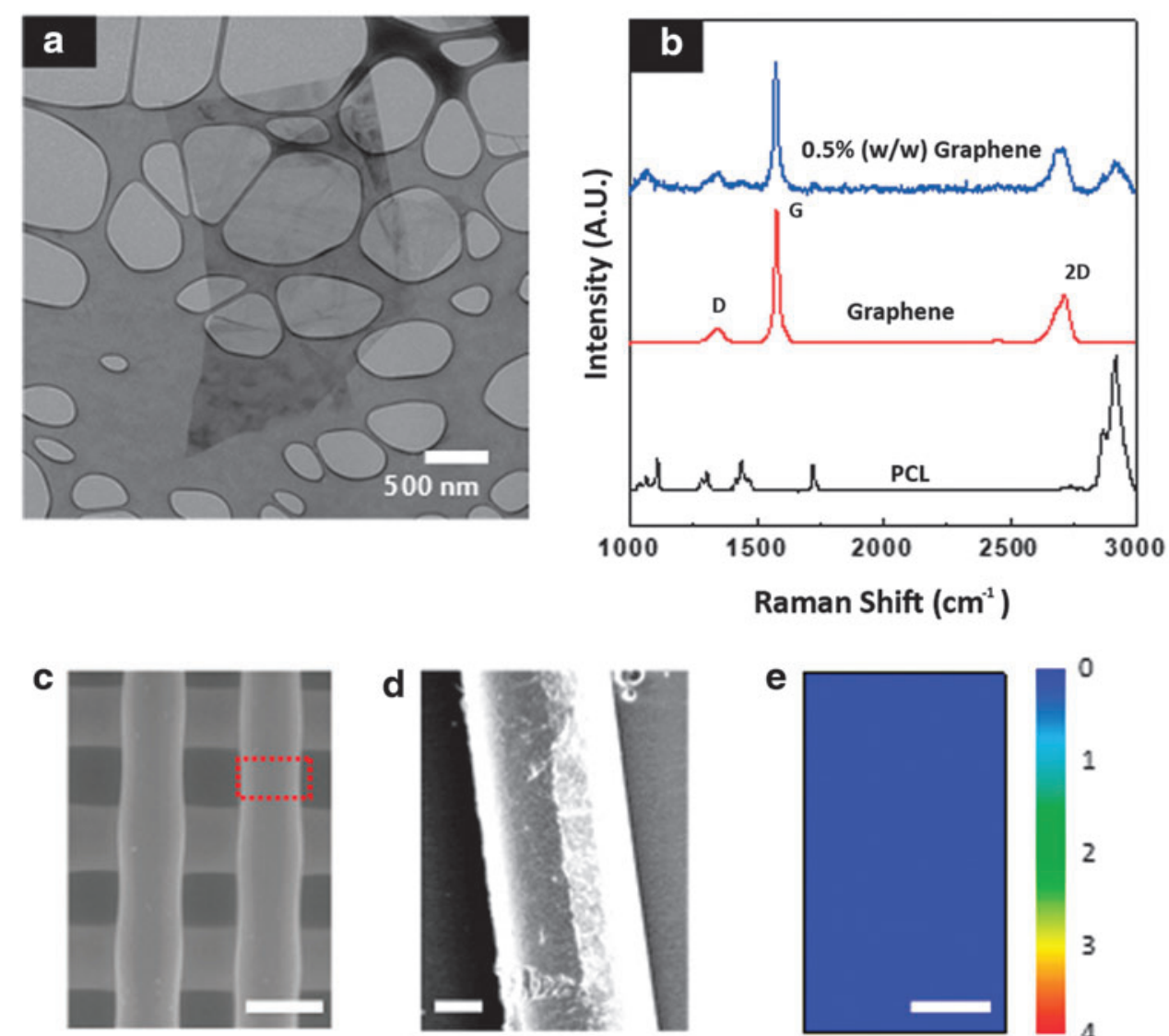

d
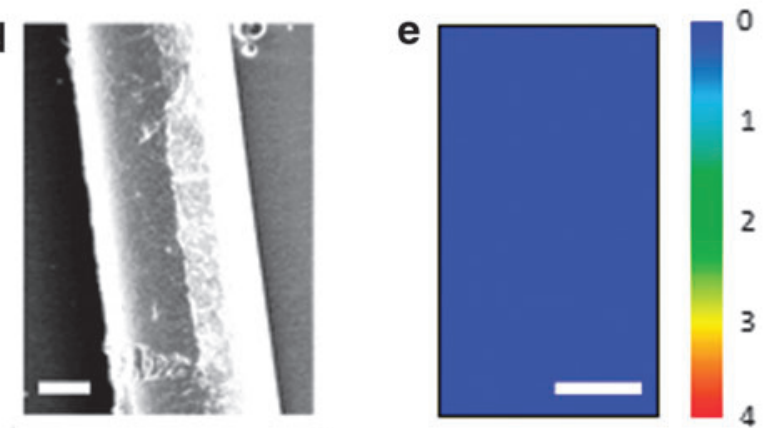

f

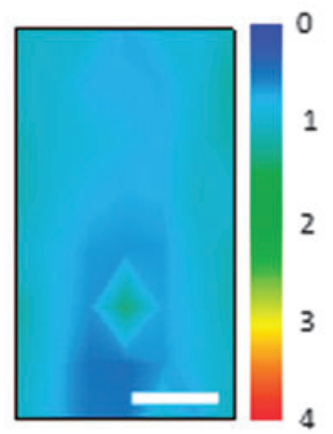

g

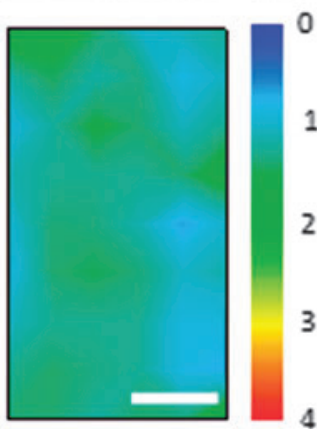

h

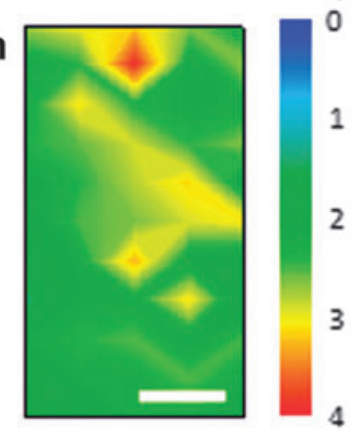

i
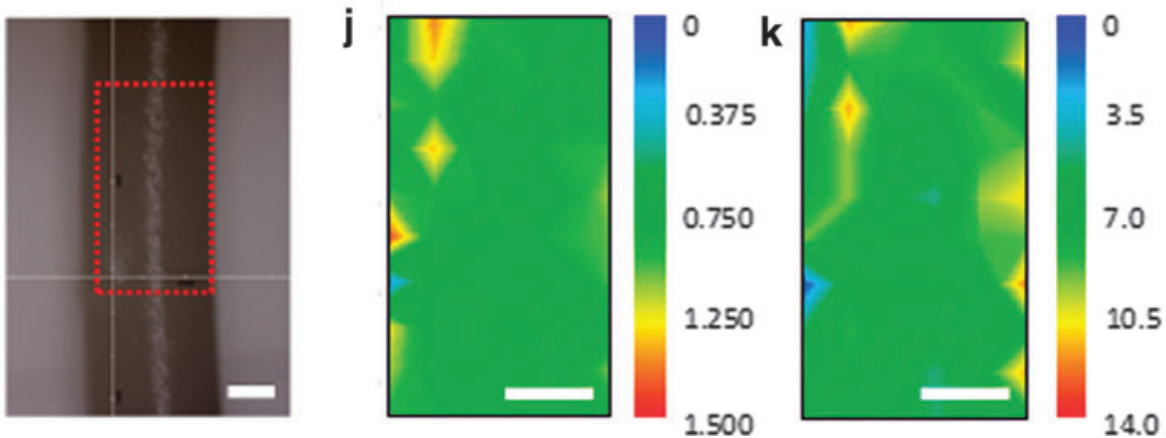

FIG. 2. (a) TEM image of graphene nanosheet. (b) Raman spectra of PCL, graphene, and PCL/graphene scaffold containing $0.50 \mathrm{wt} . \%$ of graphene. (c) SEM image of PCL/graphene scaffold $(0.50 \mathrm{wt} . \%)$. The red dotted box corresponds to the position where the Raman mapping was performed in $\mathbf{e}-\mathbf{h}$. Scale bar $=400 \mu \mathrm{m}$. (d) Magnified SEM image of PCL/graphene scaffold $(0.50 \mathrm{wt} . \%)$. Scale bar $=100 \mu \mathrm{m}$. Raman mapping of PCL/graphene scaffold with varying concentrations of graphene (e) $0 \mathrm{wt} . \%$, (f) $0.13 \mathrm{wt} . \%$, (g) $0.50 \mathrm{wt} . \%$, and (h) $0.78 \mathrm{wt} . \%$. Scale bar $=100 \mu \mathrm{m}$. (i) SEM image of PCL/graphene scaffold with 0.50 wt. \% graphene. Scale bar $=100 \mu \mathrm{m}$. Red dotted box: Raman mappings of PCL/graphene scaffold $(0.50$ wt. \%) with ratios of integrating the intensities of the $\mathrm{G}$ band from 1574 to $1580 \mathrm{~cm}^{-1}$ for graphene to the intensities of $(\mathbf{j})$ from 1435 to $1441 \mathrm{~cm}^{-1}$ peak and (k) from 2924 to $2931 \mathrm{~cm}^{-1}$ peak for PCL, respectively. Scale bar $=100 \mu \mathrm{m}$. TEM, transmission electron microscopy. Color images are available online at www.liebertpub.com/3dp 
mapping intensity indicates the ratio of integrating the intensity of the $\mathrm{G}$ band from 1574 to $1580 \mathrm{~cm}^{-1}$ for graphene to the intensity of $1435-1441 \mathrm{~cm}^{-1}$ peak for PCL. Results show uniform dispersion of graphene. Figure $3 \mathrm{~g}$ represents a small region of PCL/graphene scaffold with $0.50 \mathrm{wt}$.\% graphene considered to obtain more detailed information. The corresponding mapping intensities presented in Figure $2 \mathrm{j}$ and $\mathrm{k}$ indicate the ratios of integrating the intensities of the $G$ band from 1574 to $1580 \mathrm{~cm}^{-1}$ for graphene to the intensities of $1435-1441 \mathrm{~cm}^{-1}$ peaks and $2924-2931 \mathrm{~cm}^{-1}$ peaks for PCL, respectively. Overall, the micro-Raman mappings show uniform distribution of graphene nanosheets in the printed scaffold.

\section{Cell viability/proliferation and P1-latex protein}

Figure $3 \mathrm{a}$ shows representative images after $24 \mathrm{~h}$ of scratching. Figure $3 \mathrm{~b}$ shows the results obtained from the scratch assay on a cell monolayer experiment with the addition of P1-latex protein to the cell culture medium. The results suggest that the $\mathrm{P} 1$-latex protein has a positive impact on the cell proliferation from an early stage. A significant increase on cell migration/proliferation was observed in the medium supplement with $1 \mu \mathrm{g}$ P1-latex protein. In the case of the other two concentrations $(0.01$ and $100 \mu \mathrm{g})$, it was also possible to observe higher percentage of cell migration/ proliferation than in the basal control (medium without P1), but the results are not statistically significant within $24 \mathrm{~h}$.

Figure $3 \mathrm{~d}$ shows both the protein adsorbed by the scaffolds and the protein remaining in the solution. It also shows the level of P1 measured before and after filtration (Fig. 3c). As observed, there are no significant differences between the P1 level measured before and after filtration, and low amount of P1-latex protein was adsorbed by scaffolds.

Figure $3 e$ shows cell viability/proliferation results, based on the fluorescence intensity, measured at different time points for all scaffolds. Similar fluorescence intensity values are observed for all scaffolds at day 7 . At day 14, PCL/graphene scaffolds (0.78 wt.\%) show statistically higher cell viability/ proliferation rate than PCL scaffold. Results also show that P1latex protein-coated scaffolds allow higher cell proliferation, as indicated by higher fluorescence intensity. At day 21, P1-coated scaffolds show a better biological performance enhancing cell proliferation. In addition, results show that P1-coated scaffolds containing graphene present a better biological performance than P1-coated PCL scaffolds.

\section{Osteogenic differentiation}

Figure 4 shows the ALP activity after 7, 14, and 21 days (Fig. 4a-c, respectively). After 7 days, P1-coated scaffolds cultivated in osteogenic medium show high ALP activity, statistically different from the other three groups (scaffold in osteogenic medium; Scaffold + P1 in basal medium; scaffold in basal medium). After 14 days, P1-coated scaffolds cultivated in osteogenic medium still presented statistically higher ALP than the other groups. Uncoated scaffolds cultivated in osteogenic medium presented higher ALP than the other two groups, both of them cultivated in basal medium. Similar results are observed after 21 days in all groups, with P1-coated scaffolds presenting increased values when compared with the others. Contrary to prolifera- tion, the addition of small graphene contents seems to have no effect on cell differentiation.

\section{Cell morphology and cell spreading}

Figure 5a and $\mathrm{b}$ shows cells seeded on the scaffolds at different regions. As observed, cells were able to grow forming colonies and spreading around. Cells are well spread and long extensions are observed, with cells bridging from one filament to the other. Confocal images (Fig. 5c) show that spindleshaped cell morphology is maintained (nuclei stained blue and cell membrane red).

SEM images (Fig. 5d) and confocal microscopy images (Fig. 5e) were also acquired from scaffolds coated with P1latex protein cultivated in osteogenic medium after 28 days. As observed, hADSCs were able to undergo the differentiation process induced by the osteogenic medium. It is also possible to identify a fibrillary extracellular matrix network formed by calcium deposition. The confocal images also show a significant spread of cells on scaffolds after osteogenic differentiation, with cells showing a rounded shape rather than spindle shape with long extension.

\section{Discussion}

As observed, the screw-assisted extrusion-based system operating with optimized process parameters was able to produce scaffolds with uniform distribution of graphene nanosheets, and geometric characteristics similar to the initial designed characteristics. Moreover, the low intensity ratio of $\mathrm{D}$ to $\mathrm{G}$ band (ID/IG) and strong 2D intensity peaks suggest that the prepared graphene has high crystallinity and low defects. ${ }^{32}$

As a strong material, the addition of even small graphene levels to PCL scaffolds improved mechanical properties, with scaffolds containing $0.78 \mathrm{wt} \%$ presenting a compressive modulus close to the cortical bone. Besides improving the mechanical properties of printed scaffolds, the multifunctional nature of graphene substrates influences the behavior of different cell types, mainly due to its nanotopographic features, which provides an appropriate surface for cell attachment. As the surface properties of graphene can be controlled, this 2D material offers opportunities for cellular stimulation to maximize the desired biological response, as proposed by Dubey et al. ${ }^{33}$ and Goenka et al. ${ }^{34}$ Preliminary results suggest that the addition of graphene increases protein adsorption. $35-37$

The results presented in this article show low adsorption profiles of P1-latex protein for both PCL and PCL/graphene scaffolds. This can be explained by the hydrophobic nature of PCL, the relatively low concentration of graphene, and the high concentration of P1-latex protein in the initial solution. Nevertheless, the results show that the use of P1-latex protein has a positive impact on both cell proliferation and cell differentiation. P1-coated scaffolds containing graphene presented higher cell proliferation, which is in accordance with previous studies on the biological impact of graphene. ${ }^{38}$ The results suggest that P1-latex protein released from the scaffolds was able to directly stimulate cell proliferation along the 3D fibers of the scaffolds, displaying growth factor-like behavior. Moreover, this protein also enhances the osteogenic differentiation, as observed by measuring the ALP activity. 
a
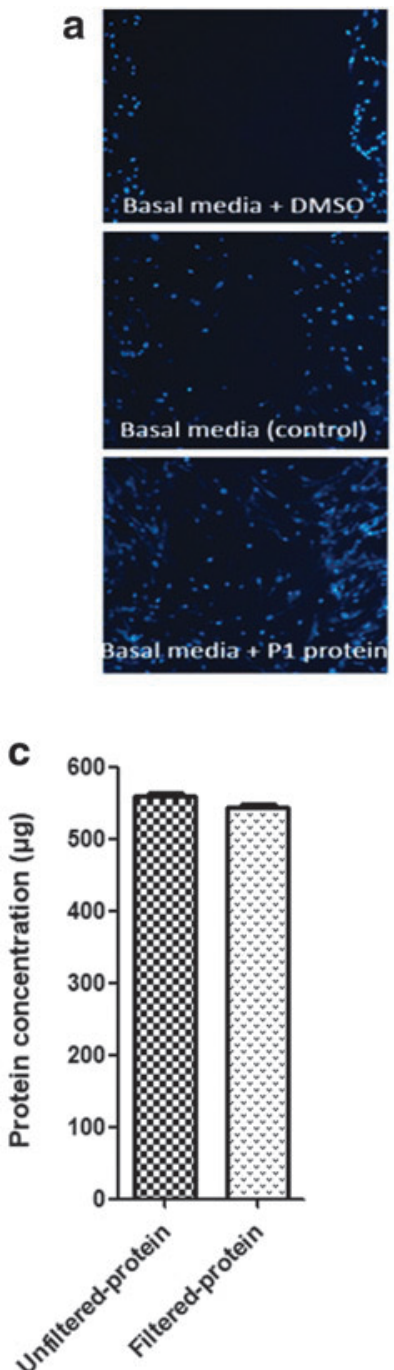

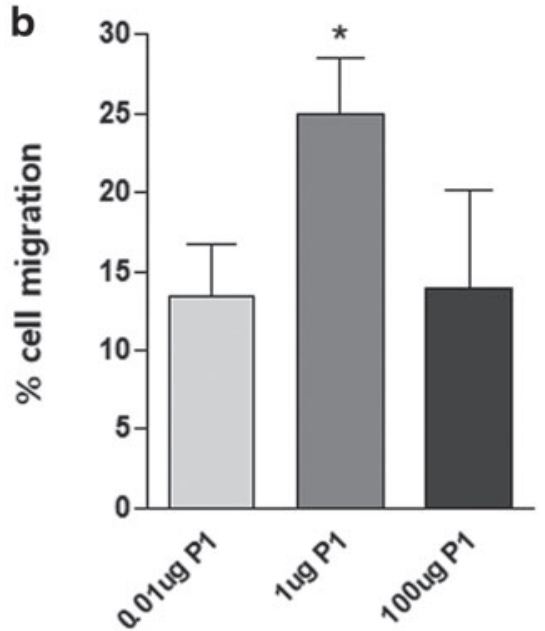

d

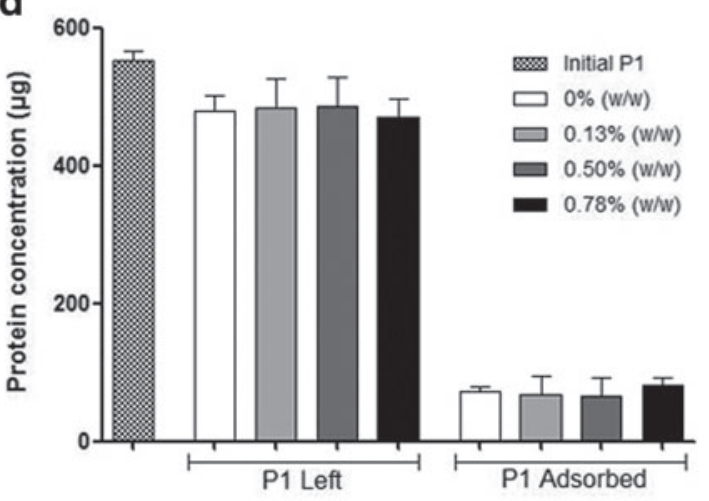

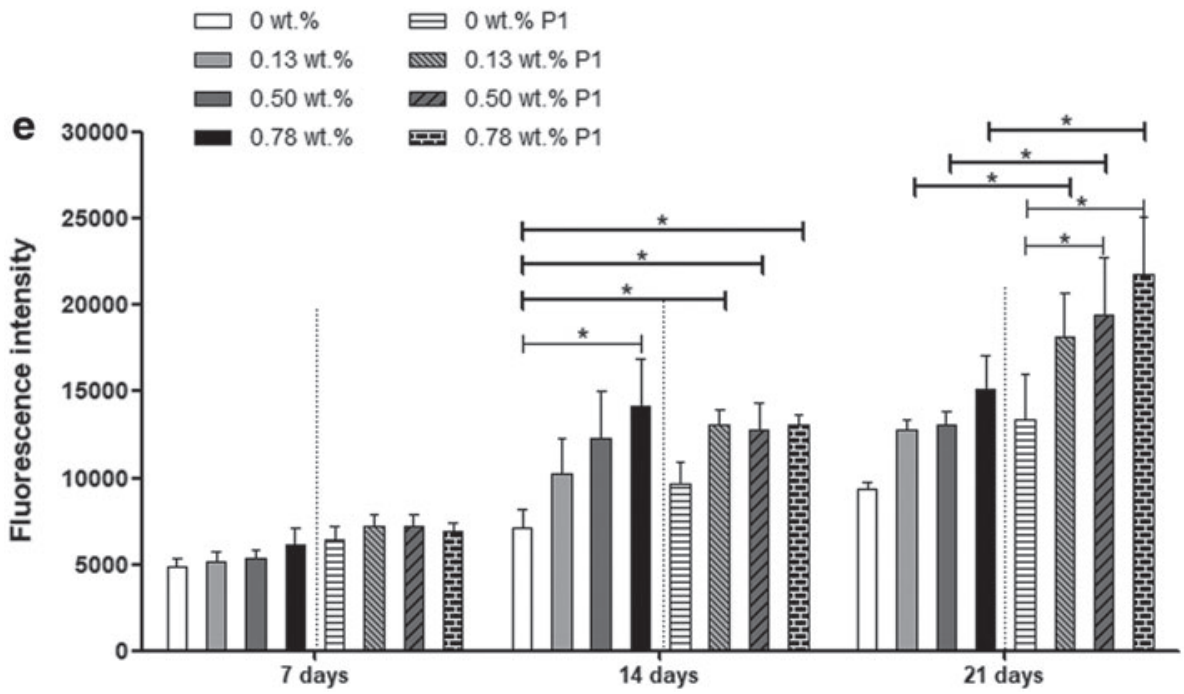

FIG. 3. hADSC monolayer migration and proliferation with P1 assay. (a) Images presenting the negative control group (basal medium + DMSO), basal medium as control group, and basal medium with $1 \mu \mathrm{g}$ P1. (b) Percentage of cell migration/proliferation related to the basal medium control group. (c) P1 protein concentration before and after filtration previously to scaffold adsorption. (d) Amount of P1 protein adsorbed by the scaffolds and remaining in the solution. (e) Alamar Blue assay. Results are expressed according to fluorescence intensity performed after 7, 14, and 21 days of cell seeding. Scaffolds uncoated with P1 protein and scaffolds coated with P1 protein were considered. Data are graphically reported as mean value and the standard deviation. Statistical analysis was performed by two-way ANOVA followed by post hoc Bonferroni test. *Statistical analysis difference $(p<0.05)$. DMSO, dimethyl sulfoxide; hADSC, human adiposederived stem cell. Color images are available at www.liebertpub.com/3dp 

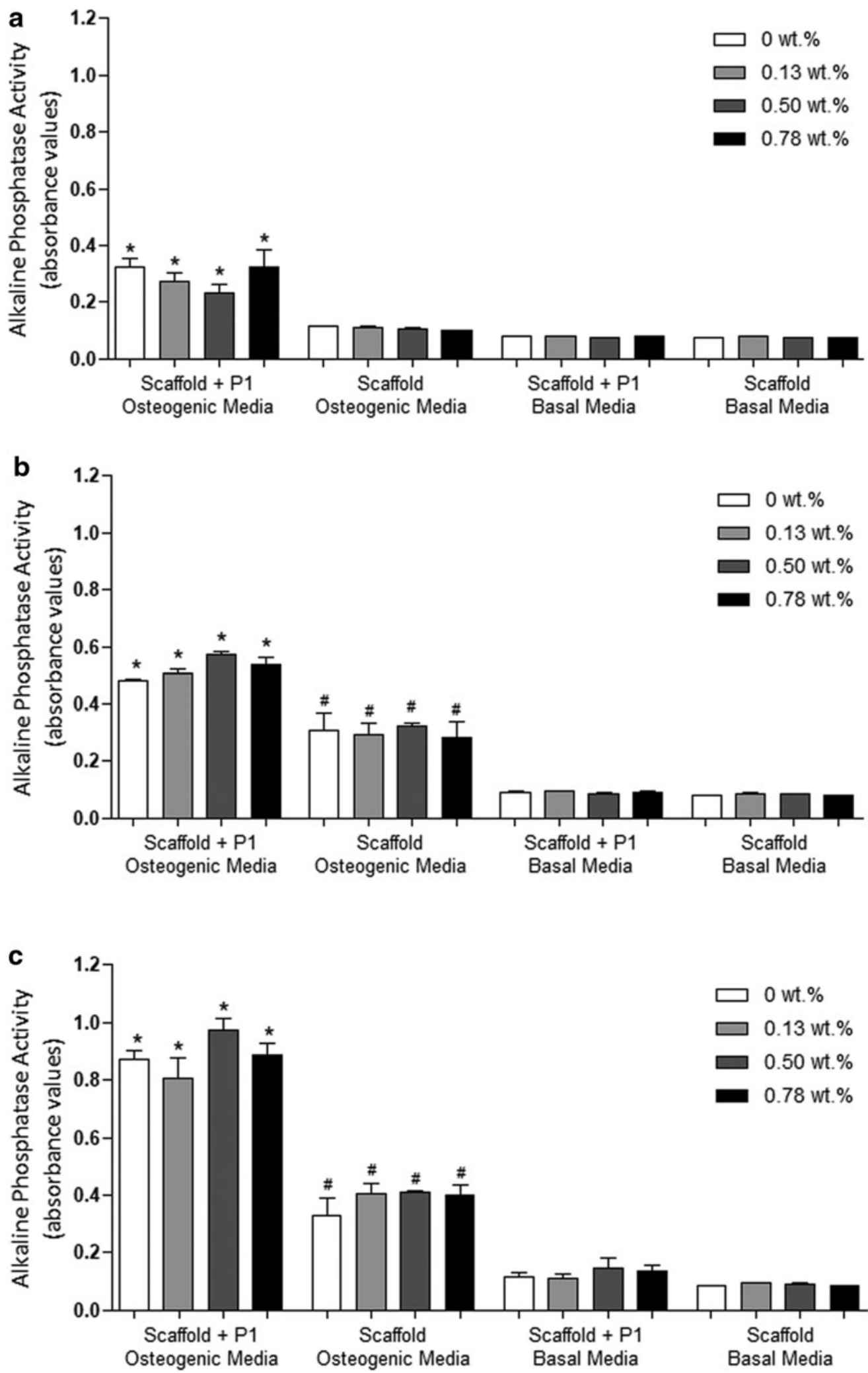

FIG. 4. Alkaline phosphatase activity measurement: (a) after 7 days, (b) after 14 days, and (c) after 21 days. Statistical analysis was performed by one-way ANOVA followed by post hoc Tukey test. *Statistical analysis difference $(p<0.05)$ among scaffold + P1 in osteogenic medium group and all other groups; " statistical analysis difference $(p<0.05)$ between scaffold in osteogenic medium group and basal medium groups. 


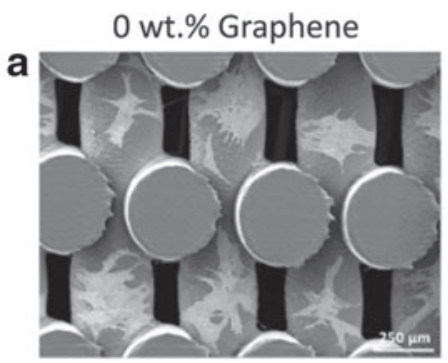

0.13 wt.\% Graphene

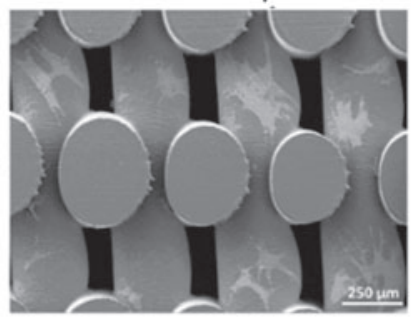

b

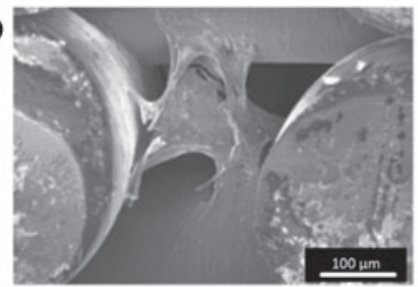

C
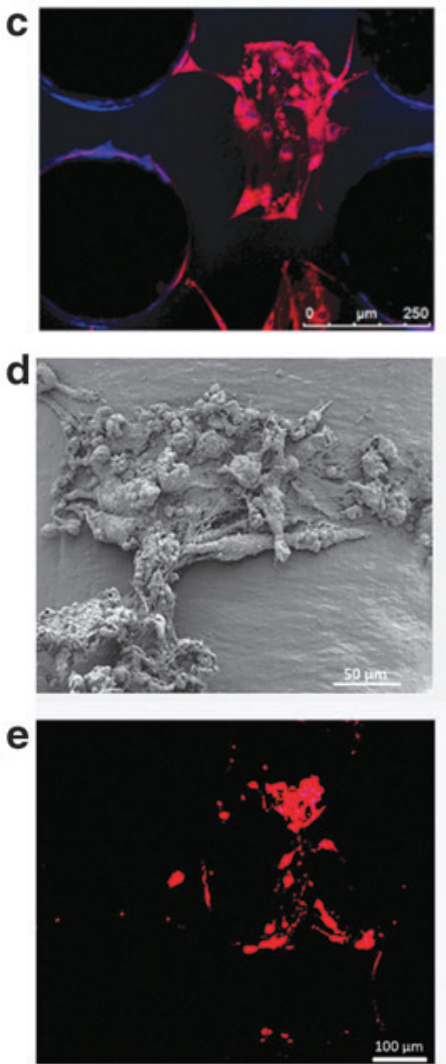
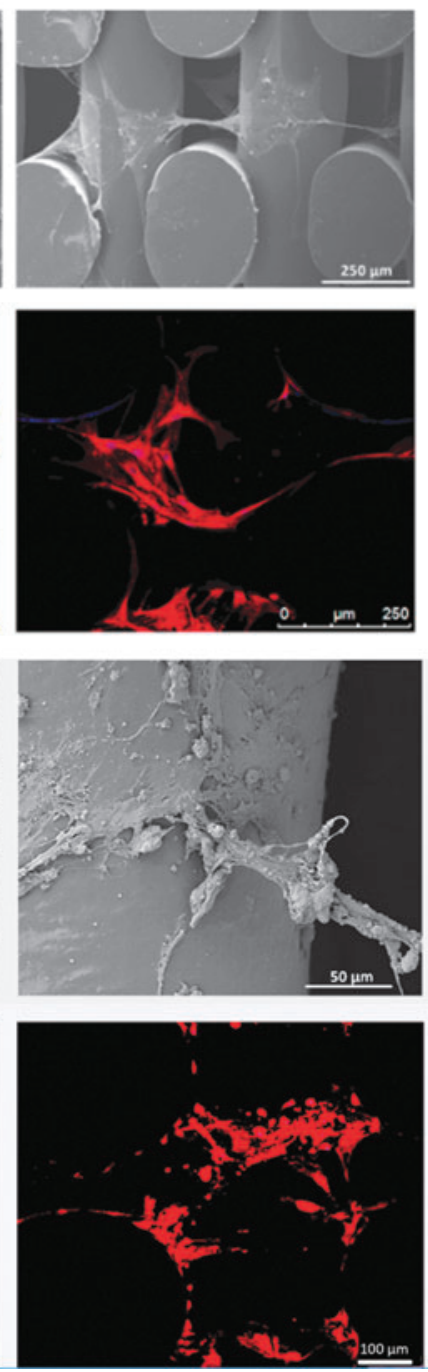

0.50 wt.\% Graphene
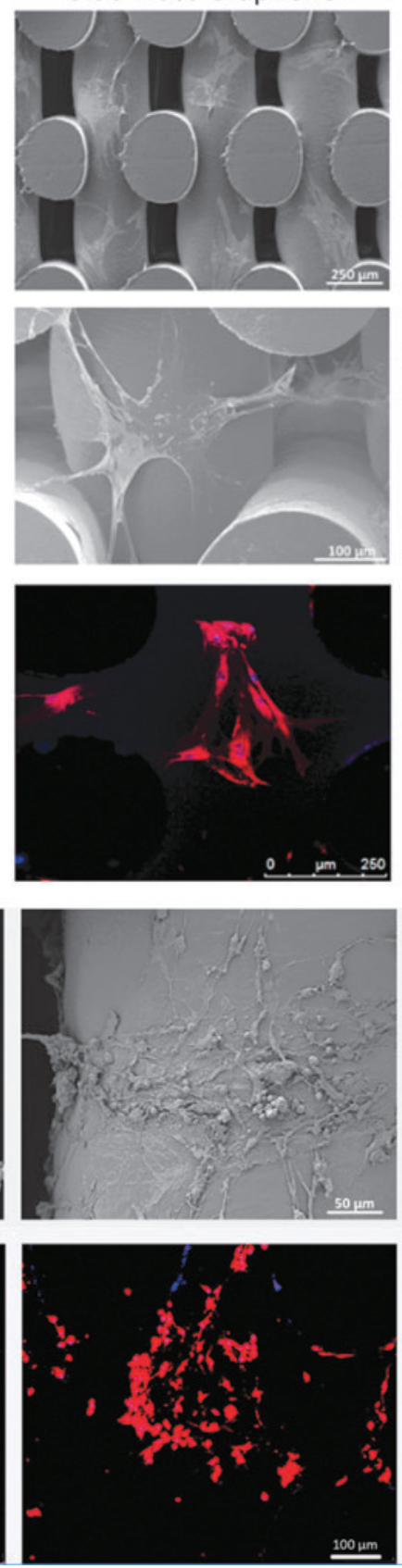

0.78 wt.\% Graphene
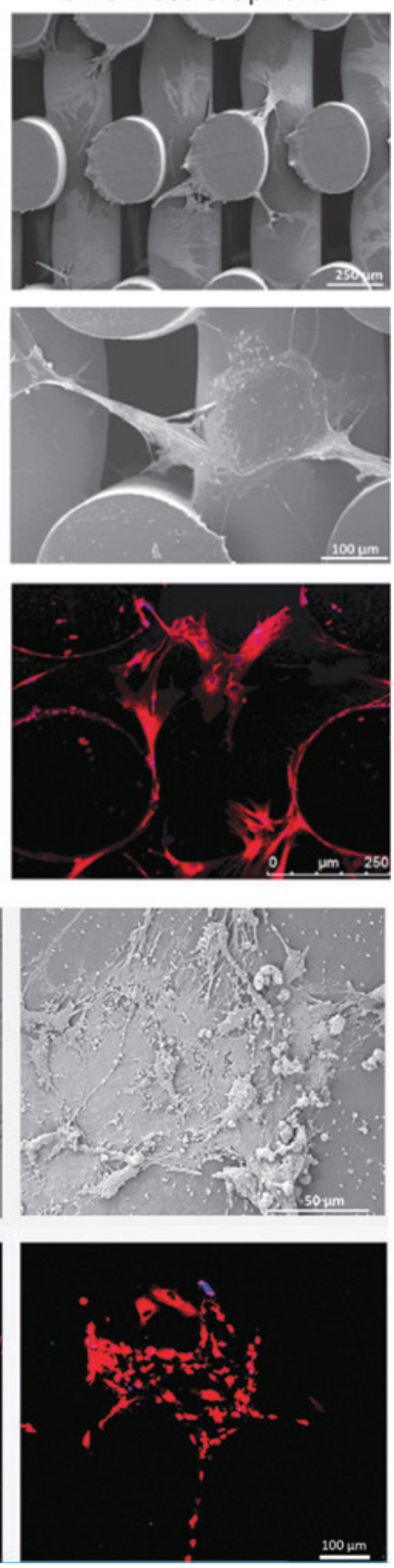

FIG. 5. Cell morphology/attachment and cell spreading on neat PCL and PCL/graphene scaffolds. (a,b) SEM images at different magnifications. (c) Laser confocal microscopy images. (d) SEM and (e) laser confocal microscopy images of cell osteogenic differentiation on PCL/graphene scaffolds after 28 days. Scaffolds coated with P1 protein. Color images are available at www.liebertpub.com/3dp

\section{Conclusions}

This study demonstrates for the first time the successful combination of additive manufacturing and protein-coating processes to produce PCL/graphene/P1-latex protein for bone regeneration with adequate mechanical properties and improved biological performance, promoting hADSCs adhesion, proliferation, and differentiation. Graphene nanosheets are well distributed in the PCL scaffold fibers, confirming that screw-assisted extrusion additive manufacturing is a viable technique to produce scaffolds with geometric characteristics (filament diameter and pore size) close to the designed characteristics. Results show that the presence of low levels of graphene (maximum $0.78 \mathrm{wt} . \%$ ) improves cell attachment and proliferation but has no significant impact on cell differentiation. Moreover, results from coated scaffolds compared with those from uncoated scaffolds show that the P1-latex protein has a positive impact on the cell biological behavior, enhancing both cell proliferation and osteogenic differentiation. The results suggest that the scaffolds investigated in this article can be used for bone regeneration. 


\section{Acknowledgments}

The authors thank the School of Mechanical, Aerospace, and Civil Engineering, University of Manchester, and Ribeirão Preto School of Medicine, University of São Paulo. Financial support was received from Fundação de Amparo a Pesquisa do Estado de São Paulo (FAPESP), Brazil (Process 2014/23662-1).

\section{Author Disclosure Statement}

The authors state there are no conflicts of interest regarding the publication of this article.

\section{References}

1. Melchels FP, Domingos MA, Klein TJ, et al. Additive manufacturing of tissues and organs. Prog Polym Sci 2012; 37:1079-1104.

2. Katari RS, Peloso A, Orlando G. Tissue engineering. Adv Surg 2014;48:137-154.

3. Mao A, Mooney DJ. Regenerative medicine: Current therapies and future directions. PNAS 2015;112:1445214459.

4. Bartolo P, Kruth JP, Silva J, et al. Biomedical production of implants by additive electro-chemical and physical processes. CIRP Ann Manuf Technol 2012;61:635-655.

5. Pereira RF, Bartolo PJ. 3D photo-fabrication for tissue engineering and drug delivery. Engineering 2015;1:91-113.

6. Vyas C, Pereira R, Huang B, et al. Engineering the vasculature with additive manufacturing. Curr Opin Biomed Eng 2017;2:1-3.

7. Guillotin B, Guillemot F. Cell patterning technologies for organotypic tissue fabrication. Trends Biotechnol 2011;29: 183-190.

8. Murphy SV, Atala A. 3D bioprinting of tissues and organs. Nat Biotechnol 2014;32:773-785.

9. Pedde RD, Mirani B, Navaei A, et al. Emerging biofabrication strategies for complex tissue constructs. Adv Mater 2017;29:1606061.

10. Pereira RF, Sousa A, Barrias CC, et al. Advances in bioprinted cell-laden hydrogels for skin tissue engineering. Biomanufact Rev 2017;2:1.

11. Khademhosseini A, Langer R. A decade of progress in tissue engineering. Nat Protoc 2016;11:1775-1781.

12. Pati F, Gantelius J, Svahn HA. 3D bioprinting of tissue/ organ models. Angew Chem Int Ed 2016;55:4650-4665.

13. Wang Y, Guo G, Chen H, et al. Preparation and characterization of polylactide/poly ( $\varepsilon$-caprolactone)-poly (ethylene glycol)-poly ( $\varepsilon$-caprolactone) hybrid fibers for potential application in bone tissue engineering. Int $\mathbf{J}$ Nanomed 2014;9:1991.

14. Di Luca A, Szlazak K, Lorenzo-Moldero I, et al. Influencing chondrogenic differentiation of human mesenchymal stromal cells in scaffolds displaying a structural gradient in pore size. Acta Biomater 2016;36:210-219.

15. Basha RY, Doble M. Design of biocomposite materials for bone tissue regeneration. Mater Sci Eng C 2015;57:452463.

16. Patrício T, Domingos M, Gloria A, et al. Fabrication and characterisation of PCL and PCL/PLA scaffolds for tissue engineering. Rapid Prototyp J 2014;20:145-156.

17. Sousa I, Mendes A, Pereira RF, et al. Collagen surface modified poly ( $\varepsilon$-caprolactone) scaffolds with improved hydrophilicity and cell adhesion properties. Mater Lett 2014;134:263-267.

18. Sasmazel HT. Novel hybrid scaffolds for the cultivation of osteoblast cells. Int J Biol Macromol 2011;49:838-846.

19. Balabanian CA, Coutinho-Netto J, Lamano-Carvalho TL, et al. Biocompatibility of natural latex implanted into dental alveolus of rats. J Oral Sci 2006;48:201-205.

20. Issa JP, Defino HL, Pereira YC, et al. Bone repair investigation using rhBMP-2 and angiogenic protein extracted from latex. Microsc Res Tech 2012;75:145-152.

21. Ereno C, Guimarães SA, Pasetto S, et al. Latex use as an occlusive membrane for guided bone regeneration. J Biomed Mater Res A 2010;95:932-939.

22. Mendonça RJ, Maurício VB, de Bortolli Teixeira L, et al. Increased vascular permeability, angiogenesis and wound healing induced by the serum of natural latex of the rubber tree Hevea brasiliensis. Phytother Res 2010;24:764-768.

23. Sampaio RB, Mendonca RJ, Simioni AR, et al. Rabbit retinal neovascularization induced by latex angiogenicderived fraction: An experimental model. Curr Eye Res 2010;35:56-62.

24. Manna K, Hsieh CY, Lo SC, et al. Graphene and grapheneanalogue nanosheets produced by efficient water-assisted liquid exfoliation of layered materials. Carbon 2016;105:551555.

25. Manna K, Huang HN, Li WT, et al. Toward understanding the efficient exfoliation of layered materials by waterassisted cosolvent liquid-phase exfoliation. Chem Mater 2016;28:7586-7593.

26. Fiedler T, Videira AC, Bártolo $\mathrm{P}$, et al. On the mechanical properties of PLC-bioactive glass scaffolds fabricated via BioExtrusion. Mater Sci Eng C 2015;57:288-293.

27. Patel JJ, Modes JE, Flanagan CL, et al. Dual delivery of epo and bmp2 from a novel modular poly- $\varepsilon$-caprolactone construct to increase the bone formation in prefabricated bone flaps. Tissue Eng Part C Methods 2015;21:889-897.

28. Fronza M, Heinzmann B, Hamburger M, et al. Determination of the wound healing effect of Calendula extracts using the scratch assay with $3 \mathrm{~T} 3$ fibroblasts. J Ethnopharmacol 2009;126:463-467.

29. Poh PS, Hutmacher DW, Holzapfel BM, et al. In vitro and in vivo bone formation potential of surface calcium phosphate-coated polycaprolactone and polycaprolactone/ bioactive glass composite scaffolds. Acta Biomater 2016; 30:319-333.

30. Borra RC, Lotufo MA, Gagioti SM, et al. A simple method to measure cell viability in proliferation and cytotoxicity assays. Braz Oral Res 2009;23:255-262.

31. Wu S, Liu X, Yeung KW, et al. Biomimetic porous scaffolds for bone tissue engineering. Mater Sci Eng R Reports 2014;80:1-36.

32. Cancado LG, Pimenta MA, Neves BR, et al. Influence of the atomic structure on the Raman spectra of graphite edges. Phys Rev Lett 2004;93:247401.

33. Dubey N, Bentini R, Islam I, et al. Graphene: A versatile carbon-based material for bone tissue engineering. Stem Cells Int 2015;2015:12.

34. Goenka S, Sant V, Sant S. Graphene-based nanomaterials for drug delivery and tissue engineering. J Control Release 2014; 173:75-88.

35. Kumar S, Chatterjee K. Comprehensive review on the use of graphene-based substrates for regenerative medicine and biomedical devices. ACS Appl Mater Interfaces 2016;8: 26431-26457. 
36. Akhavan O, Ghaderi E, Shahsavar M. Graphene nanogrids for selective and fast osteogenic differentiation of human mesenchymal stem cells. Carbon 2013;59:200-211.

37. Lee WC, Lim CH, Shi H, et al. Origin of enhanced stem cell growth and differentiation on graphene and graphene oxide. ACS Nano 2011;5:7334-7341.

38. Wang W, Caetano G, Ambler WS, et al. Enhancing the hydrophilicity and cell attachment of 3D printed PCL/graphene scaffolds for bone tissue engineering. Materials 2016;9:992.
Address correspondence to:

Paulo Bártolo

Manchester Biomanufacturing Centre School of Mechanical, Aerospace and Civil Engineering

University of Manchester Manchester M13 9PL

United Kingdom

E-mail: paulojorge.dasilvabartolo@manchester.ac.uk 